\title{
Impacts Des Innovations Sur Les Facteurs De Production Dans Le Département De Nioro : Cas Des Semoirs Double Boîte
}

\author{
Awa Dioum \\ Etudiante à l'école Doctorale Développement Durable et Société, l'université \\ de THIES, Senegal \\ Pr. Samba Arona Ndiaye \\ Chef de department production végétale à l'Ecole Supérieur Nationale \\ d'agriculture, Thies,Senegal

\section{Souleymane Dia} \\ Maître de Conférences à l'Ecole Supérieure d'Economie Appliquée, \\ Université Cheikh Anta Diop, Dakar, Senegal
}

\section{Doi:10.19044/esj.2019.v15n11p145 URL:http://dx.doi.org/10.19044/esj.2019.v15n11p145}

\section{Résumé}

Situé dans la région de Kaolack, le département de Nioro du Rip est le cœur du bassin arachidier. À l'image du reste du pays, la majorité de sa population travaille dans l'agriculture, activité qui est fortement dépendante de la pluviométrie perturbée depuis plus de quatre décennies par la variabilité climatique. À cela s'ajoute un système de production archaïque. Aujourd'hui, face à un défi d'adaptation d'un système de production dans le contexte de $\mathrm{CC}$, une innovation agricole est mise au point. Elle se traduit essentiellement par l'introduction des semoirs double boîte. À cet égard, l'objectif de cette étude est de voir les impacts de cette innovation sur les facteurs de production que sont : le travail, la main d'œuvre et les intrants. Ainsi, une enquête directe est faite auprès des producteurs et des observations directes sont faites sur le terrain. Ces observations ont permis de bien apprécier l'utilisation des SDB (semoirs double boîte). L'exploitation et l'interprétation démontrent des impacts positifs sur le facteur de travail et sur les facteurs agro économiques. Aussi, des impacts sociaux marqués par la protection sanitaire des producteurs et l'allégement du travail des femmes sont notés.

Mots-clés: Semoir à double boîte, Microdosage, Facteur travail, Impact agro économique, Nioro du Rip 


\title{
Impact of Innovations on Production Factors in the Department of Nioro : Cases of Double Box Seeders
}

\section{Awa Dioum}

Etudiante à l'école Doctorale Développement Durable et Société, l'université de THIES, Senegal

\author{
Pr. Samba Arona Ndiaye
}

Chef de department production végétale à l'Ecole Supérieur Nationale d'agriculture, Thies, Senegal

Souleymane Dia

Maître de Conférences à l'Ecole Supérieure d'Economie Appliquée, Université Cheikh Anta Diop, Dakar, Senegal

\begin{abstract}
Located in the Kaolack region, the Nioro department is the heart of the peanuts zone. Like other areas of the country, the majority of its population is involved in agriculture. This activity is highly dependent on rainfall, which has been disrupted by climate variability for more than four decades. In addition, there is an archaic production system. Today, faced with the challenge of adapting a production system in the vicinity of CC, an agricultural innovation is being developed. This is mainly reflected in the introduction of double gearbox seed drills. This paper focuses on understanding the impacts of this innovation on the following factors of production: work, labor, and inputs. Thus, a direct survey has been conducted with farmers through questionnaires and direct observations made in the field. These observations have made it possible to evaluate the use of the SDB. An analysis and interpretation of the results have shown significant impacts on labor input. Agro-economic impact is also observed due to the positive effects of microdosing. Finally, there are social impacts marked by the health protection of producers and the reduction of women's work.
\end{abstract}

Keywords: Double box planter, Microdose, Labor factor, Agro-economic impact, Nioro

\section{Introduction}

L'agriculture est un facteur essentiel de croissance économique. Selon la Banque Mondiale, elle contribue à 3,54\% du PIB du monde. Au Sénégal, elle occupe plus de $70 \%$ de la population active et participe au 16,047\% du 
PIB (Banque Mondiale, 2017). Toutefois, cette agriculture est assujettie durant ces deux dernières décennies aux effets des changements climatiques aussi bien qu'à un système agricole peu productif. En fait, les transformations agricoles qui se sont déroulées à la fin du XXIe siècle reposaient sur une intensification à grande échelle nécessitant une grande quantité d'intrants (FOA, 2017). Ceci à contribuer à aggraver les conséquences sur l'environnement. Pourtant, dans un contexte de grande incertitude, selon les variations structurelles (changement climatique etc.) et des conditions de vie des sociétés, ces dernières doivent se montrer créatives et réinventer les modalités de production, de transformation des produits agricoles dans une perspective à long terme qui tienne en compte les territoires et les communautés qui y vivent, tout en focalisant l'attention sur le concept de durabilité (GIORDANO, 2015).

Dans le département de Nioro, en plus de la dégradation des conditions biophysiques, les producteurs sont confrontés à l'usage d'un système de production archaïque. Ce système se traduit par l'utilisation de matériels rudimentaires et des pratiques culturales qui mobilisent une main d'œuvre importante et de grandes quantités d'intrants, la plupart des outils de travail utilisés comme le semoir traditionnel datant des années 60 (Harvard et al., 1996). Toutefois, les paysans réagissent en adaptant leurs techniques et pratiques agricoles qui ne sont plus adaptées aux conditions actuelles de production.

C'est dans ce contexte que les semoirs double boîte sont introduits. Ces dits semoirs, sont très importants dans le sens où ils doivent permettre une optimisation des facteurs de production. Par facteurs de production nous comprenons le travail, la main d'œuvre et les intrants.

Par ailleurs, cette innovation peut être considérée comme un des facteurs clefs de l'augmentation de la productivité des terres et du travail (Duru et al., 2014).

Dans ce sens, l'objectif de cette étude est donc d'étudier les impacts des semoirs double boîte sur les facteurs de production. Plus spécifiquement, il s'agira de mesurer leur impact sur le temps de travail pour les semis et l'application des engrais, sur la main d'œuvre et sur les opérations de fertilisation des sols.

\section{MÉTHODOLOGIE}

\subsection{Présentation de la zone d'étude}

Le département de Nioro du Rip est limité au Nord par celui de Kaolack, au Sud par la République de Gambie, à l'Est par la région de Kaffrine et à l'Ouest par la région de Fatick. Il s'étend sur une superficie de $2302 \mathrm{~km}^{2}$, soit $42,97 \%$ du territoire régional. Il est traversé par la route nationale $\mathrm{n}^{\circ} 4(\mathrm{RN} 4)$ qui mène vers la République de la Gambie et les régions situées au sud du pays. 


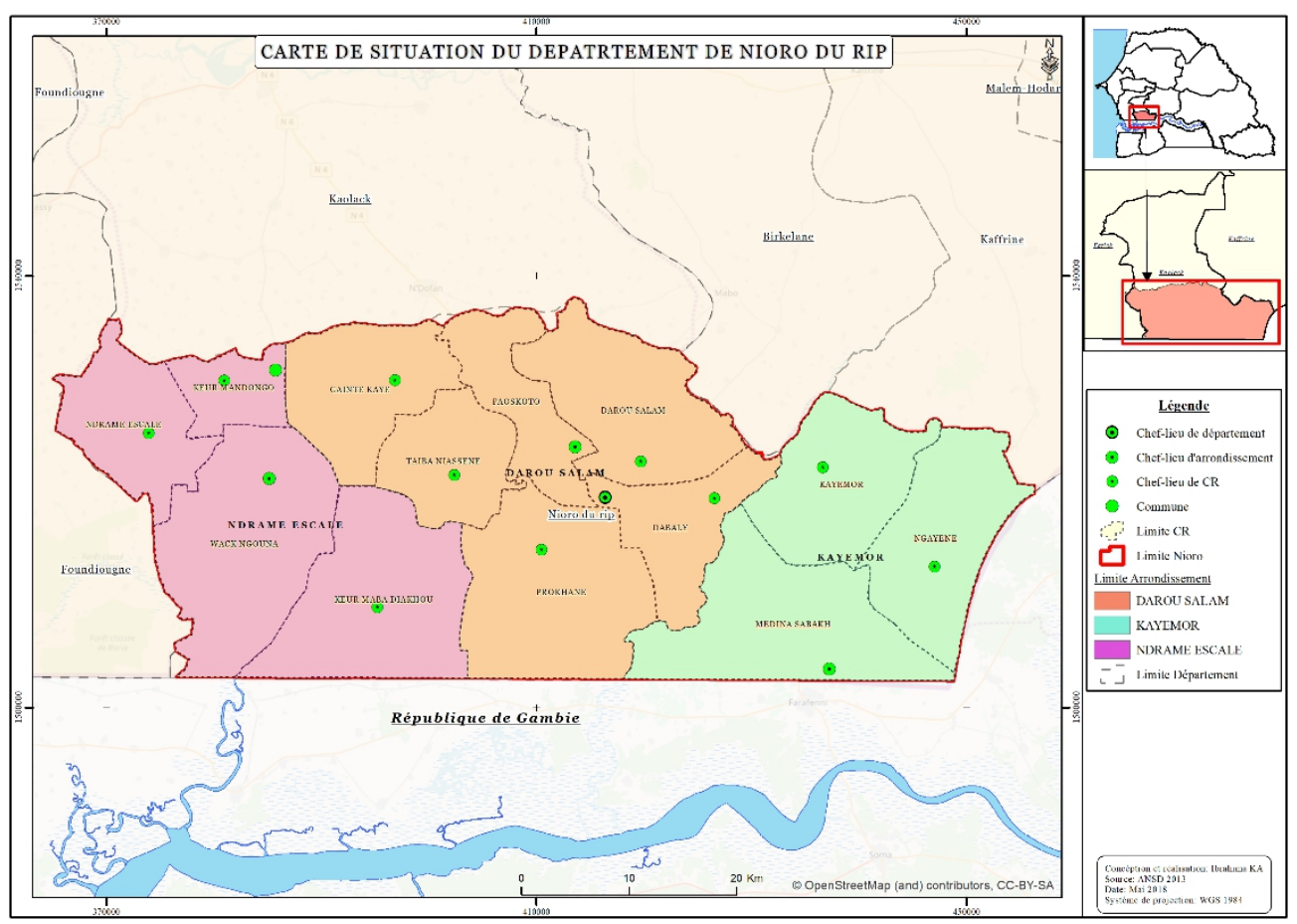




\subsection{Méthode et technique d'échantillonnage}

Du fait que notre option méthodologique est axée sur la méthode quantitative, l'échantillonnage est basé sur la technique stratifiée aléatoire simple. Pour cela, l'exploitation des données secondaires existantes est utilisée pour définir la taille de l'échantillon.

\subsection{Taille de l'échantillon}

Compte de l'étendu de la zone d'étude, un zonage a été fait suivant les critères suivants : pédologie, type d'érosion, la végétation et l'hydrographie. Ainsi 3 zones sont identifiées. Puis, pour définir la taille de l'échantillon, deux options sont choisies. D'abord, l'utilisation des données secondaires existante. Ce qui a permis de d'avoir une base de $\mathbf{1 1 2 7}$ producteurs suivant des critères d'appartenance à la zone d'étude, l'activité agricole, la socio-économie et la taille des exploitations. Puis, un pourcentage de $\mathbf{2 5 , 3 8 \%}$ est appliqué. Ainsi, l'étude portera sur $\mathbf{2 8 7}$ producteurs qui sont répartis de manière proportionnelle dans les 3 zones identifiées avec le zonage suivant le nombre de producteur identifié dans chaque zone. Enfin, les villages sont choisis au hasard dans chaque zone de même que les producteurs.

Tableau 1. Répartition de la taille de l'échantillon par zone

\begin{tabular}{|c|c|c|c|}
\hline Zones & $\begin{array}{c}\text { Nombre producteur } \\
\text { par zone }\end{array}$ & Pourcentage & $\begin{array}{c}\text { Nombre de producteurs à } \\
\text { enquêter }\end{array}$ \\
\hline Zone 1 & 827 & 73 & 210 \\
\hline Zone 2 & 258 & 23 & 65 \\
\hline Zone 3 & 42 & 4 & 11 \\
\hline Total & 1127 & & $\mathbf{2 8 6}$ \\
\hline
\end{tabular}

Source : Dioum, 2017

- Formule pour la répartition des producteurs enquêtés.

$\mathrm{N}=$ nombre total des producteurs

$\mathrm{X}=$ représente le nombre de producteurs de chaque zone dans $\mathrm{N}$

$\mathrm{E}=$ effectif total des producteurs à enquêter (taille de l'échantillon)

$\mathrm{Npz}=$ effectif des producteurs par zone.

Ainsi : $\mathrm{Npz}=\mathrm{X}^{*} \mathrm{E} / 100$

\subsection{Les outils et techniques de collecte de données}

Essentiellement des questionnaires et des guides d'entretien sont utilisés. Les questionnaires sont administrés directement aux producteurs et les guides d'entretien aux acteurs du département comme l'ISRA (Institut Sénégalais de Recherches Agricoles) et le CPA (Centre de Promotion Agricole), etc. Pour faire le zonage, des cartes d'occupation du sol et des infrastructures sont utilisées. 
De même, pour la collecte des données la technique d'entretien semistructuré et des observations directes sont effectuées.

\section{RÉSULTATS}

\subsection{Impact positif sur le facteur travail}

L'impact sur le travail est analysé à deux niveaux : la main d'œuvre et le temps de travail.

\subsubsection{Nette baisse de la main d'œuvre}

À partir de la lecture de la Figure 1, une baisse considérable de la main d'œuvre est constatée. En fait, 50\% de la main est réduite avec l'utilisation du semoir double boîte. Ceci est observé au niveau des 3 zones.

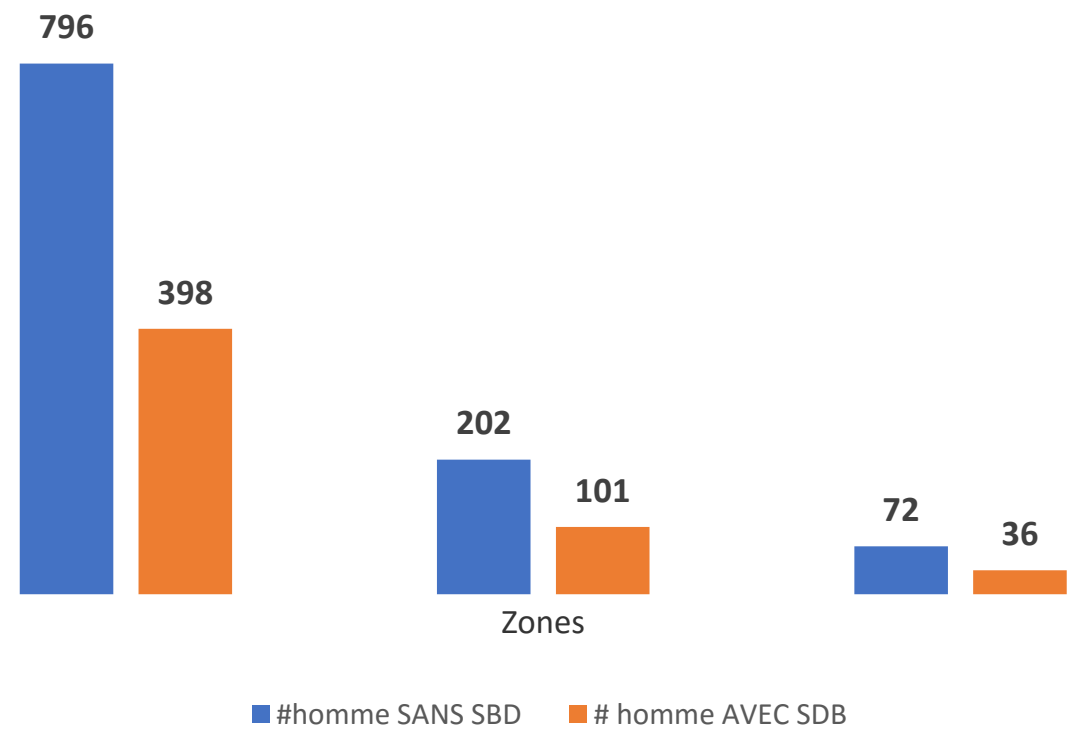

Figure 1 : Réduction Baisse de la main d'œuvre par zone selon le type de semoir Source : Dioum, 2017

En outre, la faible mécanisation et la concentration du calendrier agricole sur une période limitée expliquent l'intensité du travail pendant la saison active. La rétro-comparaison avec les pratiques antérieures montre que le SDB réduit la main d'œuvre. Ceci s'explique par la possibilité de faire le semis et l'épandage d'engrais au même moment avec au plus 2 hommes/jour pour une superficie d' 1 ha. Ces deux activités se faisaient sur un intervalle de 10 jours et l'épandage d'engrais à lui seul mobilisait presque toute la famille y compris les femmes. En outre, cette réduction entraîne un gain financier important qui est évalué à $50 \%$ du montant investit avec les semoirs traditionnels 1284000F CFA. 

travail.

La réduction de la main d'œuvre va de pair avec celle du temps de

\subsubsection{Baisse du temps de travail}

Le temps de travail dans le secteur agricole est de 8h/jour. Avec les semoirs traditionnels, le temps de travail est évalué à 20,5heures pour 3 jours de travail. Ici nous avons considéré la réalité de zone qui fait que la majorité des producteurs enquêtés travaille de $7 \mathrm{~h}$ à $12 \mathrm{~h}$, soit 4 heures de temps de travail par jour.

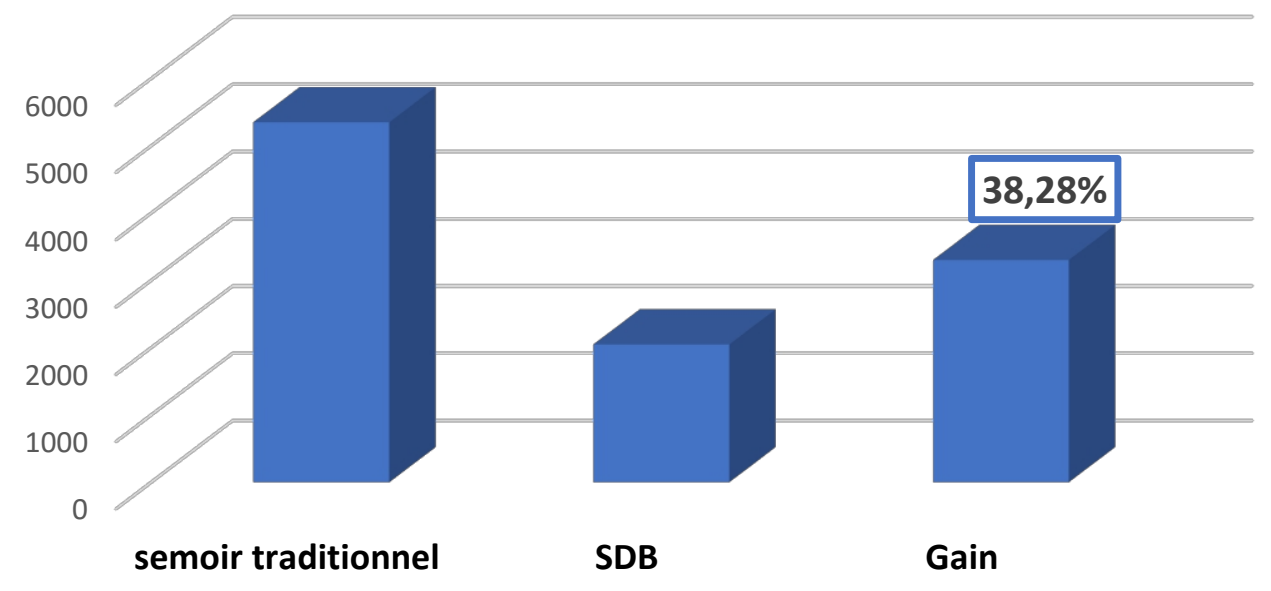

Figure 2: Réduction du temps de travail selonle type de semoir Source : Dioum, 2017

La figure 2 montre un gain considérable sur le temps de travail. À l'échelle du département, l'usage des semoirs double boîte ont permis une économique de temps de 38,28\% sur 5350 heures avec les semoirs traditionnels. Par ailleurs, ce gain de temps est important pour les femmes qui pour seulement l'épandage d'engrais pouvaient travailler sur plus de 3000 heures à l'échelle du département. En fait, dans le département de Nioro les femmes et les enfants sont les principaux acteurs pour l'activité de l'épandage d'engrais. Selon elles, le gain de temps permettra d'autres occupations sociales comme les regroupements.

En plus de la réduction du facteur travail, l'usage des SDB permet une optimisation des intrants, en particulier les engrais, avec la possibilité d'application du microdosage. 


\subsection{Impacts agro- économiques}

2.2.1. Importance du microdosage

Avec les semoirs double boîte, le microdosage devient une réalité chez les producteurs. Le microdosage consiste à une application à petites doses à coté de chaque poquet. C'est une technique qui permet d'éviter le gaspillage de l'engrais dans la mesure où l'engrais est mis uniquement pour la plante et non pour les mauvaises herbes. En effet avec l'épandage à la volée, les mauvaises herbes profitent aussi de l'engrais et cela favorise leur développement, ce qui implique plus de travail pour le désherbage. Parfois les producteurs ne disposant pas de moyens matériels et humains voient leurs champs envahis par les herbes ce qui entraîne des abandons de parcelle. Cela constitue un frein pour l'augmentation des rendements. Selon les enquêtés, avec le microdosage, « une application unique est distribuée à la plante et cela lui permet un bon développement» et corrélativement entraine une amélioration des rendements agricoles.

Photo 1: Engrais en microdose sur le Mil

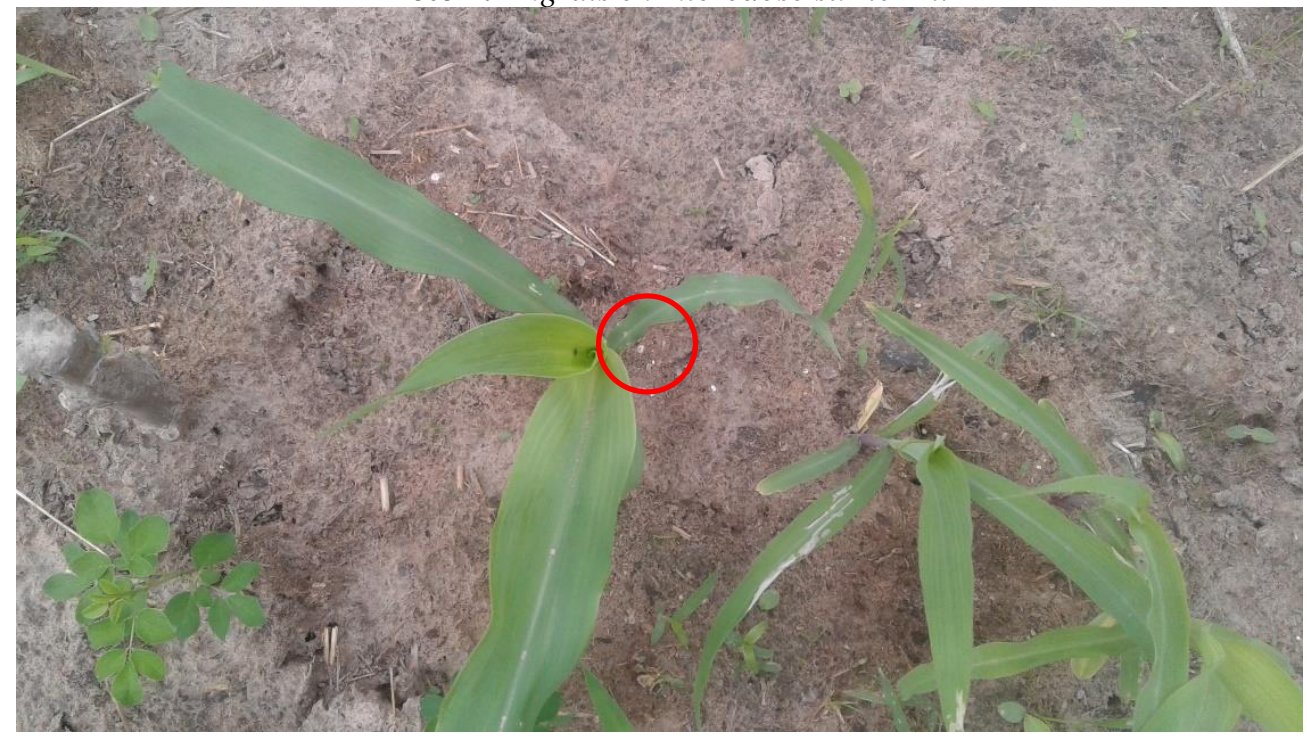

Source : Dioum, 2017

Par ailleurs, l'application de l'engrais au même moment que le semis, permet de donner de la vigueur à la levée. De ce fait, en cas de pause pluviométrique, cela minimise les pertes occasionnées par l'application sur sol sec qui réduit la bonne absorption et par conséquence la qualité de l'apport chimique. Ainsi, le microdosage rendu plus facile par le semoir double boîte entraîne une baisse des quantités d'engrais. 
2.2.2. Baisse de la quantité d'engrais

L'usage d'engrais chimique a de fortes conséquences sur l'environnement. Avec le semoir double boîte, la réduction de la quantité d'engrais est avérée. En effet, avec la technique de microdosage, le producteur utilise moins d'engrais pour $1 \mathrm{ha}(75 \mathrm{~kg}$ NPK) contre $150 \mathrm{~kg} /$ ha pour ceux qui utilisent le semoir traditionnel.

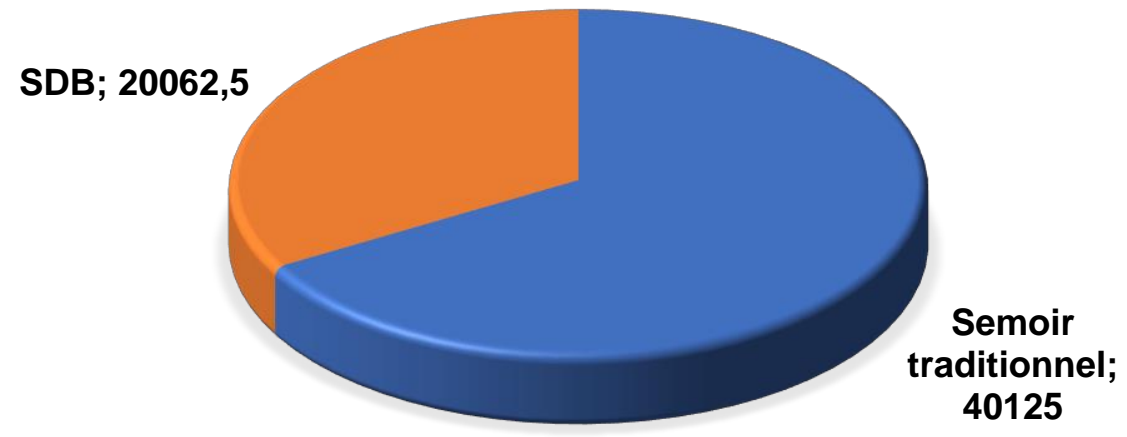

Figure 3: Quantité d'engrais selon le type de semoir Source : Dioum, 2017

Au regard de la Figure3, on note une réduction de $50 \%$ de la quantité d'engrais qui est utilisée avec les semoirs traditionnels. En fait, avec ces dits semoirs, l'épandage d'engrais est fait à la volée, ce qui ne permet pas de maîtriser le dosage de l'engrais. Aussi, il faut noter qu'avec les anciennes pratiques, les producteurs ne disposaient pas d'informations sur les besoins de fertilisant des cultures.

Dans les pratiques antérieures, pour un champ de mil il faut en moyenne $150 \mathrm{~kg}$ de NPK. A l'échelle du département, la quantité d'engrais utilisée pour une campagne hivernale pour le mil est estimée à $40125 \mathrm{~kg}$. Cette quantité sera plus importante pour l'arachide et le maïs qui sont plus exigeants en ce qui concerne la quantité de fertilisants.

En plus de l'impact agronomique, la baisse de la quantité d'engrais entraîne une réduction des montants investis pour la fertilisation.

2.2.3. Économie sur les investissements de fertilisants

Dans le contexte de dégradation des terres les producteurs utilisent beaucoup d'engrais pour augmenter les rendements. Ainsi, avec les semoirs 
double boîte, l'optimisation des engrais garanti une augmentation des rendements mais surtout une baisse des investissements pour la fertilisation chimique.

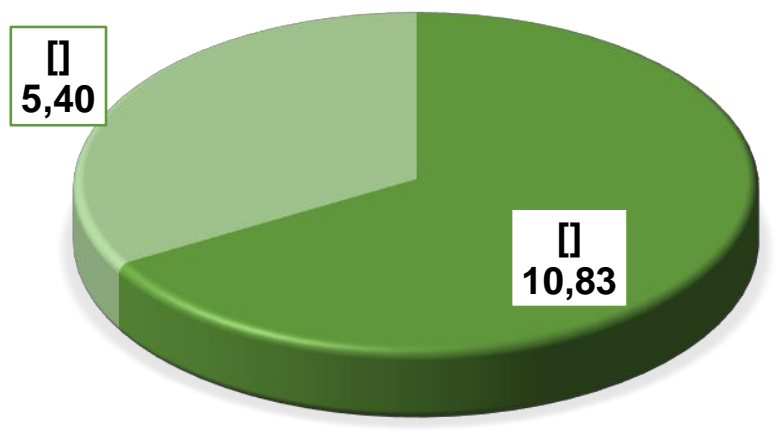

Figure 4: Investissements de fertilisation(en million de FCFA) selon le type de semoir Source : Dioum, 2017

La figure 4 montre une réduction des investissements qui passent de plus de dix million (10) à cinq million (5) de FCFA soit une baisse de 50\%.

Sous l'angle du microdosage, l'usage du SDB se révèle doublement positif dans la mesure où il permet une optimisation des intrants tout en favorisant une baisse sensible des investissements. Cette économie est utilisée selon les producteurs pour étendre les activités de production mais également cela leur permettra de prendre en charge les besoins de base de leurs familles (éducation, santé etc.). Ce faisant, il renforce aussi la sécurité alimentaire, les conditions de vie et montre par ailleurs une dimension sociale assez inattendue des impacts.

\subsection{Impacts sociaux : Santé des producteurs et allègement du travail des femmes}

La dynamique d'intensification des cultures dans le département de Nioro à travers l'utilisation d'engrais suivant les anciens pratiques a des conséquences sur la santé des producteurs et surtout celle des femmes. En fait, ces dernières sont présentes à plus de $75 \%$ pour l'activité d'épandage de l'engrais et peuvent travailler pendant 8 heures de temps par jour sous une mauvaise posture. Aussi, la manipulation manuelle de l'engrais a des 
conséquences sur la santé des producteurs, étant donné que ces derniers ne prennent pas en compte a priori les normes d'hygiène et de sécurité.

En revanche, derrière les impacts techniques et économiques immédiatement mesurables, le semoir double boîte présente des impacts sociaux moins spectaculaires mais tous aussi importants.

Le premier est lié à la santé des producteurs. En effet, la réduction du contact directe avec le produit, la réduction des heures de travail et le changement de posture influe positivement sur la santé des producteurs, surtout des femmes. Ces dernières affirment : « l'application de l'engrais n'est plus un fardeau pour nous. Désormais, nous sommes moins présentes pour cette activité car les hommes parviennent à faire cette activité avec moins d'effort et sans risques sanitaires ». Ceci entraîne une transition vers le second impact social très important qui est l'allègement des travaux des femmes. En effet, l'utilisation du semoir double boîte permet de libérer les femmes d'une activité physique éprouvante et dévoreuse de temps, alors qu'elles n'étaient déchargées d'aucune de leurs tâches habituelles. La possibilité de cumuler le semis et l'épandage de l'engrais avec le semoir double boîte, réduit la sollicitation des femmes. Ainsi, les femmes seront plus actives dans les activités communautaires dans leur localité.

\section{DISCUSSION}

L'introduction du semoir double boîte constitue sans doute une innovation dans le contexte des changements climatiques et de maintien d'un système de production archaïque. Aujourd'hui, les agriculteurs et leurs pays ont besoin continuellement d'innover pour répondre aux demandes changeantes des marchés et de rester compétitif (BM, 2008).

Au-delà des multiples impacts positifs, attendus ou surprenants, l'intérêt du SDB est qu'il semble jeter les bases d'une possible résilience des systèmes. Cette capacité d'orientation vers la résilience est également défendue par Conway qui pense que: «tous les systèmes pluviaux ont besoin d'une technologie capable d'accroître la stabilité de la productivité et la résilience des systèmes de production » (Conway, 1999).

Par ailleurs, le semoir double aide à l'augmentation des rendements par le biais de l'optimisation des intrants et apporte un gain certain sur la productivité. Aussi, son impact agronomique à travers le microdosage est marqué par une réduction de la quantité de fertilisant qui peut avoir des conséquences sur l'environnement. Selon (Lamine, 2017), les pratiques d'intensification exercent un impact négatif sur l'environnement, sur la santé des agriculteurs et des populations et, posent aujourd'hui la question de la durabilité de ces modes de production et de consommation alimentaire.

Toutefois, à l'instar des SDB, de nombreuses technologies ont des impacts positifs sur l'environnement qui ne sont pas pris dans les bénéfices privés des 
agriculteurs qui adoptent ces technologies. Et cela pourrait être une source de motivation pour l'adoption des dites technologies. Dans cette étude, la question d'adoption ne se pose pas. En fait, cette innovation est portée par les producteurs locaux. Elle est une réponse technique aux problèmes d'adaptation des producteurs face aux problèmes d'accès aux intrants, à la dégradation des conditions biophysiques dans un contexte de changement climatique. Pour nous une innovation doit apporter des solutions pratiques, applicables et appliquées par les populations locales et pouvant apporter un changement visible au niveau des acteurs au plus bas de l'échelle. De la même manière, elle ne doit pas uniquement permettre une augmentation des rendements. Selon les études de la Banque Mondiale (2008) l'innovation doit s'atteler aussi à la préservation des ressources et aux adaptations nécessaires en regard des différences entre les hommes et les femmes. Dans le cadre cette étude, il est montré que le SDB est à la fois bénéfique tant pour les hommes que pour les femmes, non seulement à travers la réduction du temps de présence des femmes mais aussi par les gains économiques qui peuvent être ressentis directement par les hommes.

C'est pourquoi il est permis de penser que lorsqu'il aura plus de vécu et qu'il sera approfondi par d'autres innovations, le SDB sera un levier possible pour construire la résilience des terroirs ruraux du Département, par la transformation des écosystèmes, l'évolution sociale, et le développement agro-économique. En ce sens, Daniel et al. (2008), affirment que : «les méthodes et les approches qui permettent d'intégrer une innovation doivent être au centre des débats afin de permettre l'appropriation par les acteurs et donc accroître l'impact des améliorations technologiques et leur pérennisation dans des contextes où les marges de manœuvre économiques et climatiques sont très réduites $»$.

\section{CONCLUSION}

L'objectif de cette étude est d'étudier les impacts du SDB sur les facteurs de production. Les résultats montrent que le SDB est une innovation technique capable d'impacter en profondeur sur un système de production. En plus des impacts positifs sur les facteurs travail et l'allégement des travaux des femmes, le SDB a un impact agro-économique marqué par les gains sur les intrants et corrélativement sur leur coût.

\section{References:}

1. Banque Mondiale (2008). Rapport sur le développement dans le monde: l'agriculture au service du développement, De Boeck University, 424p. 
2. BM (2017). La situation mondiale de l'alimentation et de l'agriculture : mettre les systèmes alimentaires au service d'une transformation rurale inclusive. 201p.

3. Conway, G. (1999). The Doubly Green Revolution : Food for All the in the Twenty First Centery, Ithaca,NY,orney University PressInternet.

4. Daniel Clavel, Barro Albert, Belay Tesfay, Lahmar Rabah, \& Maraux Florent (2008). Changements techniques et dynamique d'innovation agricole en Afrique Sahélienne : le cas du Zaï mécanisé au Burkina Faso et de l'introduction d'une cactée en Ethiopie ». Burkina fasse. $59 \mathrm{p}$ : Revue $\mathrm{N}^{\circ} 03$.

5. Baccar Ben Lamine. M (2017). Assessment of sustainability and adaptation processes of family farms in relation to groundwater and the development of the agricultural sectors : the case of Saïs plain (Morocco),215p.

6. FOA, (2017). Situation mondiale de l'alimentation et de l'agriculture.201p.

7. Duru, M., Fares, M., \& Therond, O. (2014). Un cadre conceptuel pour penser maintenant (et organiser demain) la transition agroécologique de l'agriculture dans les territoires. Cah Agric23. 12p.

8. Giordano, S. (2015). Agriculture traditionnelle et innovante : le secteur vitivinicole biologique. Une comparaison entre les Pouilles (Italie) et le Languedoc-Roussillon, thèse en géographie et aménagement de l'espace, ecole doctrale TTSD, 551p.

9. Thiec, G. \& Harvard, M. (1996). Les enjeux du marché des matériels agricoles pour la traction animale en Afrique de l'Ouest. Agriculture et développement, $14 \mathrm{p}$.

10. Tristan, D., Xavier, C., Marie-Aude, E., \& Julien, V. (2009). Systèmes de production et itinéraires techniques agricoles. Centre d'études et de prospective service de la statistique et de la prospective, $9 \mathrm{p}$. 